\title{
The double-stranded break-forming activity of plant SPO11s and a novel rice SPO11 revealed by a Drosophila bioassay
}

Yoshinori Shingu ${ }^{1,2+}$, Takeshi Tokai ${ }^{3 \dagger}$, Yasuo Agawa ${ }^{4 \dagger}$, Kentaro Toyota $^{3}$, Selina Ahmed ${ }^{3}$, Makiko Kawagishi-Kobayashi ${ }^{5}$, Akira Komatsu ${ }^{5}$, Tsutomu Mikawa ${ }^{1,2}$, Masa-Toshi Yamamoto ${ }^{4}$, Kyo Wakasa ${ }^{3^{*}}$, Takehiko Shibata ${ }^{1,2^{*}}$ and Kohji Kusano ${ }^{4^{*}}$

\begin{abstract}
Background: SPO11 is a key protein for promoting meiotic recombination, by generating chromatin locus- and timing-specific DNA double-strand breaks (DSBs). The DSB activity of SPO11 was shown by genetic analyses, but whether SPO11 exerts DSB-forming activity by itself is still an unanswered question. DSB formation by SPO11 has not been detected by biochemical means, probably because of a lack of proper protein-folding, posttranslational modifications, and/or specific SPO11-interacting proteins required for this activity. In addition, plants have multiple SPO11-homologues.

Results: To determine whether SPO11 can cleave DNA by itself, and to identify which plant SPO11 homologue cleaves DNA, we developed a Drosophila bioassay system that detects the DSB signals generated by a plant SPO11 homologue expressed ectopically. We cytologically and genetically demonstrated the DSB activities of Arabidopsis AtSPO11-1 and AtSPO11-2, which are required for meiosis, in the absence of other plant proteins. Using this bioassay, we further found that a novel SPO11-homologue, OsSPO11D, which has no counterpart in Arabidopsis, displays prominent DSB-forming activity. Quantitative analyses of the rice SPO11 transcripts revealed the specific increase in OsSPO11D mRNA in the anthers containing meiotic pollen mother cells.

Conclusions: The Drosophila bioassay system successfully demonstrated that some plant SPO11 orthologues have intrinsic DSB activities. Furthermore, we identified a novel SPO11 homologue, OsSPO11D, with robust DSB activity and a possible meiotic function.
\end{abstract}

\section{Background}

Homologous genetic recombination plays critical roles in meiosis, for genetic diversification and precise disjunction of homologous chromosomes. In the budding yeast Saccharomyces cerevisiae, meiotic recombination is initiated by chromatin locus- and timing-specific DNA double-strand breaks [1-3], which require the function of the SPO11 protein $[4,5]$. Saccharomyces cerevisiae SPO11 shares amino acid sequence homology with

\footnotetext{
*Correspondence: k3wakasa@nodai.ac.jp; tshibata@riken.jp; kusano@dgrc.kit. ac.jp

† Contributed equally

${ }^{1}$ Cellular \& Molecular Biology Laboratory, RIKEN Advanced Science Institute,

2-1 Hirosawa, Wako, Saitama 351-0198, Japan

${ }^{3}$ Department of Agriculture, Tokyo University of Agriculture, Atsugi,

Kanagawa 243-0034, Japan

Full list of author information is available at the end of the article
}

subunit A of the type II DNA topoisomerase (topoisomerase VI) from the archaeon Sulfolobus shibatae [5]. Type II topoisomerases generate double-strand breaks (DSBs) at specified sequences in the cleavage complex, an intermediate for topological DNA reactions, composed of the covalent complex of a topoisomerase subunit and the terminus of each DSB strand. As in the cleavage complex, SPO11 covalently attaches to the $5^{\prime}$ termini of a nascent meiotic DSB. SPO11 is then removed from the DNA by the endonuclease activity of Mre11 [6-9], and by the functions of the SAE2/CPT1 $[10,11]$ and RAD50 proteins [10], but not by Nbs1, the third component of the MRN complex [8,9].

SPO11 is ubiquitous in eukaryotes, including S. cerevisiae [4], the fission yeast Schizosaccharomyces pombe (Rec12) [12], the fruit-fly Drosophila melanogaster (Mei-

\section{Biomed Central}


W68) [13], the nematode Caenorhabditis elegans (T05E11.4) [14], the basidiomycete Coprinus cinereus [15] and mammals [16], and each has a single gene encoding a SPO11-orthologue. Thus, it has been assumed that SPO11 is the essential protein to introduce DSBs for the initiation of meiotic recombination in eukaryotes [see [17]].

In contrast to these organisms, searches for homologues of SPO11 or TOP6A (subunit A of topoisomerase VI) in genome databases revealed that plants have multiple SPO11-homologues: Arabidopsis thaliana has AtSPO11-1, AtSPO11-2 and AtSPO11-3 [18,19], and their homologues in indica rice (Oryza sativa L.) are OsTOP6A1 (OsSPO11A), OsTOP6A2 (OsSPO11B) and OsTOP6A3 (OsSPO11C), respectively [20]. Genetic studies revealed that efficient meiotic recombination in Arabidopsis depends on both the AtSPO11-1 [21] and AtSPO11-2 [22] gene products, which are involved in the induction of DSBs and the initiation of meiosis. In contrast, AtSPO11-3 plays a major role during somatic cell development, but not in meiosis and meiotic recombination. The loss of the AtSPO11-3 function results in abnormal endoreduplication, an extreme dwarf phenotype and deficient cell proliferation [23-25]. AtSPO11-2 and AtSPO11-3 interact with AtTOP6B (subunit B of the archaeal topoisomerase VI), as shown by two-hybrid studies [19], suggesting that they function as DNA topoisomerases. In rice, OsTOP6A1 $(O s S P O 11 A)$ is required in rice meiosis [26]. As with AtSPO11-3, overexpression studies in Arabidopsis suggested the functions of OsTOP6A3 (OsSPO11C) and OsTOP6B in stress adaptation in mitosis [20].

Genetic studies indicated that SPO11s play critical roles in DSB formation, for the initiation of meiotic recombination. In S. cerevisiae, meiotic DSB formation for meiotic recombination initiation requires the products of at least nine genes (i.e., REC102, SKI8, REC104, REC114, MEI4, MER2, MRE11, RAD50 and NBS1I $X R S 2)$, in addition to that of the SPO11 gene [See [17] for review, [27]]. However, many of these proteins are not conserved in other organisms. Although MRE11, RAD50 and XRS2 are respectively conserved in mammals as Mre11, Rad50 and Nbs1, and in Arabidopsis as AtMRE11, AtRAD50 and AtNBS1, their corresponding proteins are not required for meiotic DSB formation $[28,29]$. Instead, for meiotic DSB formation, Arabidopsis requires PRD1, which appears to be an orthologue of mammalian Mei1 [30], while D. melanogaster requires Mei-P22 [31], which has no homologue in other eukaryotes. These observations suggested that higher eukaryotes and yeasts differ in their activation control of meiotic DSB formation and in their protein requirements for SPO11 to express its DSB-forming activity.

Genetic studies demonstrated the activity of SPO11 to generate meiotic DSBs, but the biochemical function of
SPO11 is essentially unknown. We previously isolated a soluble form of AtSPO11-1, with a DNA binding activity that functions in meiosis [32], and other plant SPO11s (Y. S. unpublished observations). However, the DSB-forming activities of AtSPO11 alone, with or without an attached tag, and of the complex of AtSPO11 with a protein (PRD1) required for SPO11-dependent DSB formation have not been detected in vitro [32]. The inability to detect the DSB-forming activities of SPO11 in vitro may simply be due to the in vitro conditions chosen for the tests, the improper folding and absence of eukaryote-specific posttranslational modifications of the protein expressed within bacterial cells, the unsuccessful multimer formation, or the absence of required SPO11-interacting proteins.

To overcome these difficulties in determining whether SPO11 by itself has DSB-forming activity and which plant SPO11 candidates function in DSB formation for meiotic recombination initiation, we tried to develop an in situ assay for DSBs, by expressing a plant SPO11 in eukaryotic cells. We chose the fruit-fly, Drosophila melanogaster, in which the expressed proteins would be folded and modified under the in vivo conditions of eukaryotic cells and the chromatin is loosened during meiosis to allow the access of SPO11 to the canonical DSB sites in chromosomal DNA [33-35]. In addition, many well-established cytological and genetic techniques are available for Drosophila. The DSBs generated by the plant SPO11 were detected by in situ immunostaining, using an antibody that recognizes Drosophila phosphorylated histone $\mathrm{H} 2 \mathrm{Av}(\gamma-\mathrm{H} 2 \mathrm{Av})$. $\mathrm{H} 2 \mathrm{Av}$ is the sole Drosophila $\mathrm{H} 2 \mathrm{~A}$ variant and the functional homolog of human $\mathrm{H} 2 \mathrm{AX}$. The phosphorylated form of H2Av accumulates at DSB sites in Drosophila [36-39], as in mammals $[40,41]$. Genetic analyses of the consequences of the ectopic expression of plant SPO11s in Drosophila would provide further support for their DSB-forming activities.

Here, we report quantitative analyses of the DSBforming activities of plant SPO11s in the absence of plant SPO11-interacting proteins in Drosophila oocytes, as a bioassay system. The DSB formation in the oocytes was further confirmed by measuring the meiotic loss of the $\mathrm{X}$ chromosome, which was expected as the result of abortive DSB formation in meiosis, in the absence of authentic SPO11. In addition, using these assays, we tested three candidates of meiotic SPO11 in rice, and identified a novel SPO11 homologue, OsSPO11D, which possesses DSB-forming activity and is expressed specifically in meiotic tissue.

\section{Results}

Detection of DSB signals induced by plant SPO11s expressed in Drosophila oocytes

Since a biochemical assay was not available, in order to address the questions of whether SPO11 has DNA 
cleavage activity by itself and which rice SPO11 candidates function in DSB formation for rice meiotic recombination, at first we tested the rice SPO11 homologues, OsSPO11A OsSPO11B and OsSPO11C, which phylogenetically correlate with AtSPO11-1, AtSPO11-2 and AtSPO11-3 (which lacks meiotic function), respectively (see later section), and the novel fourth homologue, along with the Arabidopsis SPO11 homologues, AtSPO11-1 and AtSPO11-2, for the heterospecific complementation of the reduced fertility of the Arabidopsis spo11-1 mutant. Only AtSPO11-1 complemented the deficiency of the mutant. These negative results and the advantages of the Drosophila systems as described prompted us to test Drosophila meiotic recombination mutants, to address the above questions.

A cytological technique to detect DSBs is available in Drosophila, by the use of an antibody against a phosphorylated human histone H2A variant, $\gamma-\mathrm{H} 2 \mathrm{AX}$, in which serine 139 is phosphorylated upon DSB formation $[40,42,43]$. The anti-human $\gamma$-H2AX antibody recognizes $\gamma$-H2Av, the phosphorylated Drosophila histone H2A variant, H2Av, which is phosphorylated after DSB formation induced either exogenously or during meiosis [36-38]. This technique revealed that in wild type Drosophila, DmSPO11 (Mei-W68)-dependent DSBs appeared transiently in region $2 \mathrm{~b}$ of the germarium, in which the $\gamma$-H2Av signals overlap with the chromosomal DNA signals and disappear in region 3 (stage 1) [see ref. [37] about regions and stages]. In postmeiotic nuclei of stage 3 or later egg chambers, the chromosomes are condensed to form a compact karyosome.

In yeast, unrepaired Spo11-dependent meiotic DSBs reportedly accumulated in DSB-repair defective mutants, such as rad50S, and the DSB-repair defective mutants facilitated the sensitive mapping and reliable quantification of the SPO11-dependent meiotic DSB formation $[3,44,45]$. In DSB-repair deficient mutant flies (SPO11-proficient), such as mus301, spn-A (encoding a RAD51 homologue), spn-B (encoding another RAD51 homologue) and okr (encoding a RAD54 homologue), unrepaired meiotic DSBs accumulate and persist in the oocyte nuclei of the stages beyond the germarium region $2 \mathrm{~b}$ of ovaries $[37,38]$, and cause defective karyosome morphology (partially compact karyosome) in stage 3 or later egg chambers [46]. Thus, this DSB-repair defective phenotype provides a sensitive and reliable measure of SPO11-dependent meiotic DSBs.

In our positive control experiment, mei- $W 68^{1}$ heterozygous (DmSPO11-proficient phenotype) and mus301 hemizygous (mus301-defective phenotype) flies showed the extensive accumulation of $\gamma-\mathrm{H} 2 \mathrm{Av}$ signals in almost all oocyte nuclei at stage 2-8 egg chambers, and defective karyosome morphology in most oocyte nuclei at stage 3-
8 egg chambers (100\% and $91 \%$, respectively, Figure $1 \mathrm{~A})$. The $\gamma-\mathrm{H} 2 \mathrm{Av}$ signals in the oocyte nuclei were red outside the partially compact karyosome, and some yellow signals (indicating overlap with DNA) were present in a tiny area within the stained DNA (green) area (Figure 1A and 1C). This slight overlap of the DSB signals and the major DNA signals was previously reported: while the normal karyosome in DNA repair-proficient oocytes is condensed in stage 3 or later and visualized by nuclear staining (green), in the oocyte nuclei of the DSB repairdeficient mutant $s p n-A$, a rad51 paralogue, the unrepaired DNA is dispersed and not visible by nuclear staining, except for a tiny overlapping region (yellow). However, the unrepaired DSBs were recognized by the anti- $\gamma-\mathrm{H} 2 \mathrm{AX}$ antibody (red) as $\gamma-\mathrm{H} 2 \mathrm{Av}$ foci outside the partially condensed karyosome, where the unrepaired chromosomal DNA is dispersed in the nuclei [37].

In the negative control experiment, only small fractions of the oocyte nuclei of the mei-W6 $68^{1}$ homozygous and mus301 hemizygous flies without the transgene showed DSB signals and defective karyosome morphology $(7.4 \%$ and $1.3 \%$, respectively, Figure $1 \mathrm{~B}$ and Figure $2 \mathrm{~A})$. When the DmSPO11 transgene was expressed under the Drosophila hsp 83 promoter in the mei-W68 (dmspo11) mus301 double mutant flies, almost all of the oocyte nuclei showed the extensive accumulation of $\gamma$ $\mathrm{H} 2 \mathrm{Av}$ signals, as in the case of the positive control (the DmSPO11-proficient and mus301-defective mutant), and the defective karyosome morphology $(100 \%$ and $70 \%$, respectively, Figure $1 \mathrm{C}$ ). Thus, we are confident that the $\gamma-\mathrm{H} 2 \mathrm{Av}$ signals in the postmeiotic nuclei of fly oocytes represent the DSBs formed by SPO11.

We then attempted to detect the DSBs induced by a plant SPO11, expressed in Drosophila mei-W68 ${ }^{1}$ homozygous (dmspo11-deficient) and mus301 hemizygous (mus301-defective) double mutant female flies, by an immuno-assay for phosphorylated histone H2Av $(\gamma-$ $\mathrm{H} 2 \mathrm{Av}$ ), and by monitoring the number of oocyte nuclei with defective karyosome morphology. The AtSPO11-1 or AtSPO11-2 transgene was expressed under the control of the Drosophila hsp83 promoter in the mei-W68 (dmspo11) mus301 double mutant flies. Both AtSPO11-1 and AtSPO11-2 are required for meiotic recombination in Arabidopsis $[21,22]$. Thus, we were surprised to find that the expression of either AtSPO11-1 or AtSPO11-2 significantly increased both kinds of DSB signals in flies, the frequency of $\gamma-\mathrm{H} 2 \mathrm{Av}$ signals and the defective karyosome morphology, as compared with the negative control without the transgene (Figure 1D and 1E; Figure 2A and 2B). These results indicated that both AtSPO11-1 and AtSPO11-2 have the ability to induce DSBs, without the coexpression of other plant proteins, in Drosophila oocyte nuclei.

To confirm the expression of transgenes in Drosophila oocytes, we performed a quantitative RT-PCR analysis. 


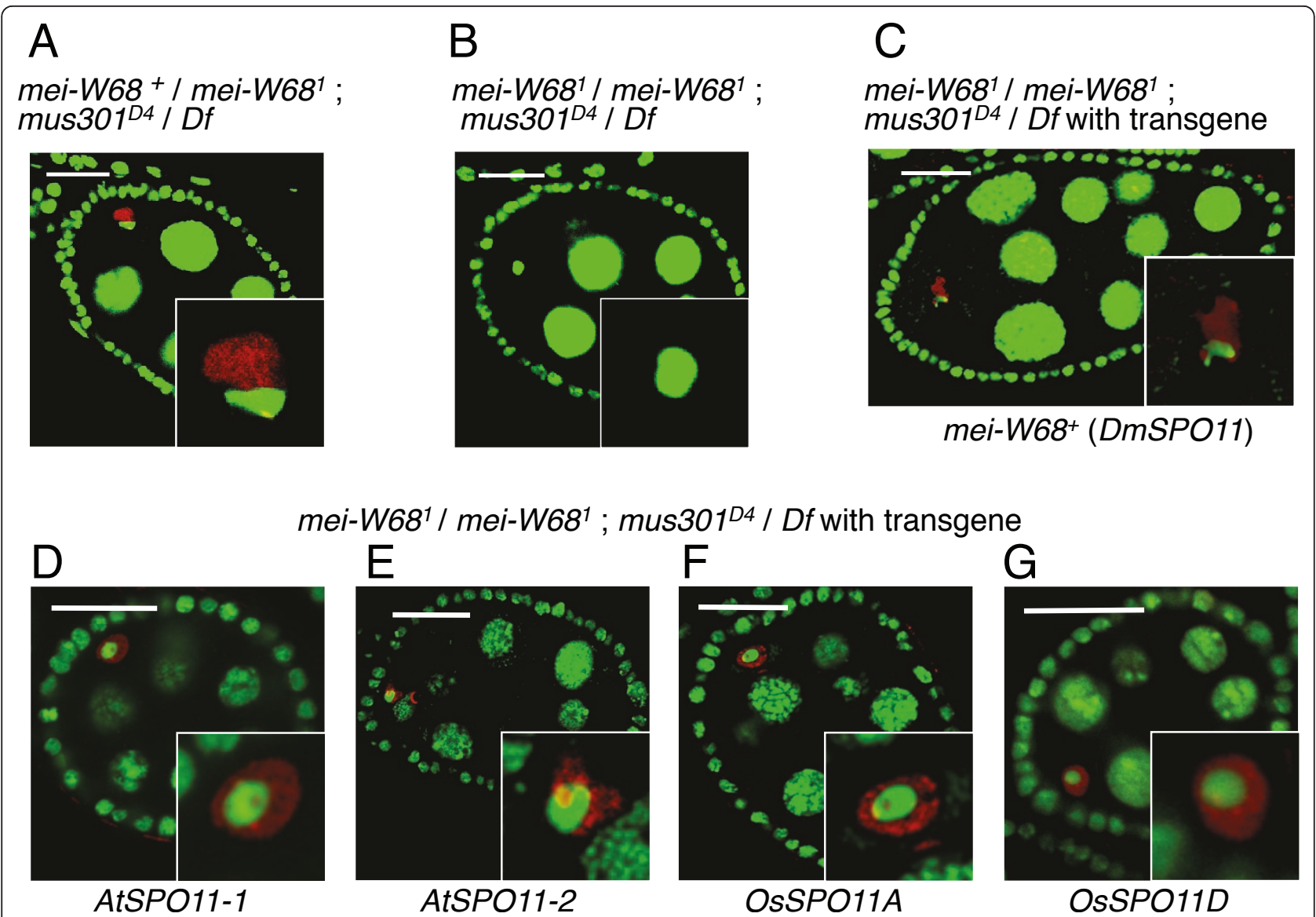

Figure 1 Confocal images of DSB signals generated by plant SPO11 expression in the mei-W68 mus301 double mutant background (mei-W68 $/$ mei-W68 $^{7} ;$ mus301 ${ }^{\mathrm{D4}} / \mathrm{Df}(3 \mathrm{~L}$ )66C-G28). The DNA is green. The $\gamma-\mathrm{H} 2 \mathrm{Av}$ is red, and shows the DSB signals. When the two signals overlap, the signal is yellow. Each inset shows an enlarged view of the oocyte nucleus. All images are single confocal sections. Each scale bar

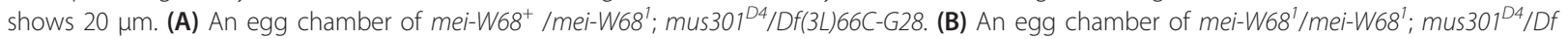
(3L)66C-G28. The frequencies of the oocyte nuclei with DSB signals for the total oocyte nuclei of stage 2-8 egg chambers were 100\% (344/345, 3 ovaries) in the mei-W68 ${ }^{\prime}$ heterozygote, and 7.4\% (16/220, 2 ovaries) in the mei-W68 homozygote (see Figure 2A). The frequencies of the oocyte nuclei with a karyosome morphological defect (Figure 1) for the total oocyte nuclei of stage 3-8 egg chambers were 91\% (254/278, 3 ovaries) in the mei-W68 heterozygote, and 1.3\% (2/177, 2 ovaries) in the mei-W68 homozygote (see Figure 2B). (C) An egg chamber of mei-W68 $/$ mei-W68 $^{1}$ mus301 ${ }^{D 4} / D f(3 L) 66 C-G 28$ with transgene P\{hsp83-mei-W68 ${ }^{+}$CDNA, M53-3\} (indicated as Vector name\{gene, insertion \#\}). The frequency of the oocyte nuclei with DSB signals for the total oocyte nuclei of stage 2-8 egg chambers was 100\% (180/180, 2 ovaries) in the mei-W68 homozygote with $P\left\{\right.$ hsp83-mei-W68 ${ }^{+}$CDNA, M53-3\}, similar to the mei-W68 ${ }^{1}$ heterozygote. The frequency of the oocyte nuclei with defective karyosome morphology for the total oocyte nuclei of stage 3-8 egg chambers was 70\% (93/132) in the mei-W68 homozygote with P\{hsp83-mei-W68 ${ }^{+}$CDNA, M53-3\}, similar to the mei-W68 ${ }^{7}$ heterozygote. The karyosomes in the insets of panels A and C (SPO11 positive oocyte with DSB repair deficiency, as positive controls) have defective morphology (non-disc shape), while the karyosome in the inset of panel B (DSB-repair defective oocyte

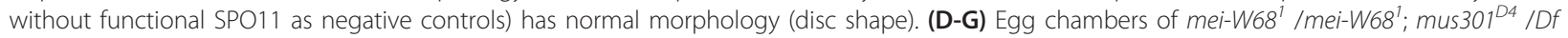
(3L)66C-G28 with transgene: P\{hsp83-AtSPO11-1 CDNA, A5-1\} (D); P\{hsp83-AtSPO11-2 CDNA, 2M3-1\} (E); P\{hsp83-OsSPO11A cDNA, 3M2-3\} (F); P\{hsp83OsSPO11D CDNA, F2-1\} (G). Quantitative data with respect to the DSB signals and the defective karyosome morphology in the oocyte nuclei of these transgenic flies are shown in Figure 2.

The expression levels of the transgenes were higher than that of the Drosophila ribosomal protein 49 (RP49) gene in Drosophila oocytes (Table 1).

Analyses of disjunction in Drosophila mei-W68 (dmspo11)deficient mutants expressing the SPO11 transgene

In order to confirm that the expressed plant SPO11 alone introduced DSBs in the chromosomes of Drosophila oocyte nuclei, we examined the profiles of $\mathrm{X}$ chromosome disjunction caused by mei-W68 (dmspo11) deficiency in the Drosophila DSB-repair proficient (mus301-proficient) background, assuming that the unrepaired DSBs caused the X chromosome loss, and the DSBs formed by the plant SPO11 restored the normal X chromosome disjunction in the dmspo11-deficient background.

The recessive null mutation of mei-W68 (dmspo11) causes female sterility associated with a high frequency 


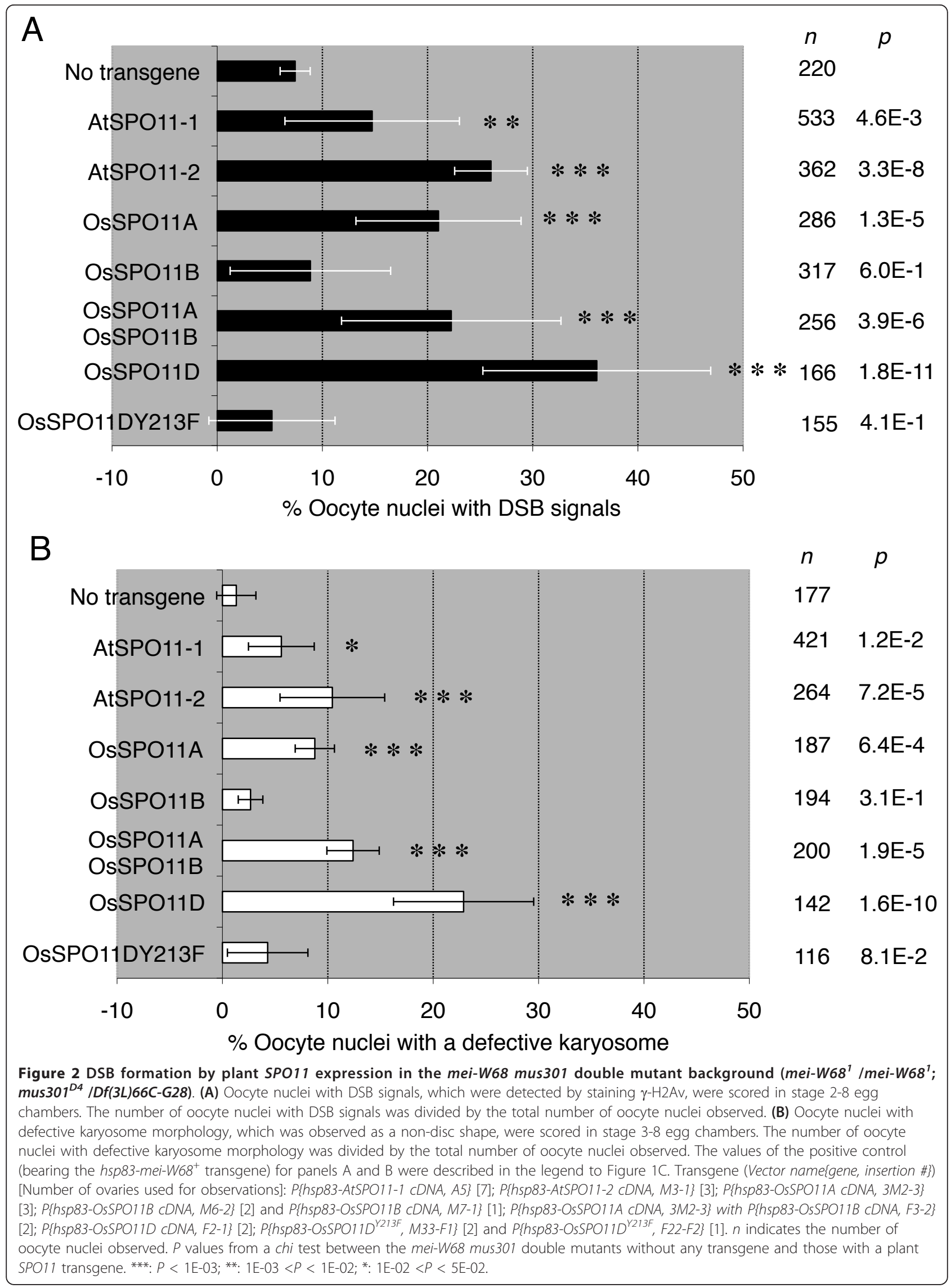


Table 1 Relative levels of mRNA from copies of the plant SPO11 transgene in Drosophila ovaries

\begin{tabular}{|c|c|}
\hline Insertions of transgenes & Relative levels of mRNA* \\
\hline P\{hsp83-AtSPO11-1 cDNA, A5-1\} & $3.40 \pm 0.40$ \\
\hline P\{hsp83-AtSPO11-2 cDNA, 2M3-1\} & $9.22 \pm 2.72$ \\
\hline P\{hsp83-OsSPO11A cDNA, 3M2-3\} & $13.88 \pm 3.59$ \\
\hline P\{hsp83-OsSPO11B cDNA, M6-2\} & $19.85 \pm 4.50$ \\
\hline P\{hsp83-OsSPO11B cDNA, F3-2\} & $14.57 \pm 6.32$ \\
\hline P\{hsp83-OsSPO11D cDNA, F2-1\} & $3.17 \pm 0.36$ \\
\hline P\{hsp83-OsSPO11D $\left.{ }^{Y 213 F}, F 22-F 2\right\}$ & $4.22 \pm 0.84$ \\
\hline P\{hsp83-OsSPO11D $\left.{ }^{Y 213 F}, M 33-F 1\right\}$ & $3.76 \pm 1.39$ \\
\hline
\end{tabular}

* The amount of mRNA from each copy of the plant SPO11 transgene in Drosophila ovaries was normalized by the amount of mRNA from the RP49 gene. The average value with SD for each of the relative levels of transcripts was obtained from three samples, each containing $5 \sim 10$ ovaries.

of chromosome nondisjunction in meiosis I, since the crossing-over type of homologous recombination induced by SPO11 is essential for chiasma formation (Figure 3A) [47]. Thus, during meiosis I in mutant cells devoid of meiotic recombination, in half of the cases, one daughter cell receives both paired chromosomes, and the other has none (i.e., nondisjunction, Figure 3B). Drosophila mei-W68 ${ }^{1}$ heterozygous (DmSPO11-proficient) mus301-proficient females displayed $88 \%$ fertility, and in contrast, mei-W68 ${ }^{1}$ homozygous (dmspo11-deficient) mus301-proficient females exhibited $26 \%$ fertility (Table 2). The ubiquitous transcription of the $m e i-W 68^{+}$ cDNA by the $h s p 83$ promoter complemented the reduced fertility of the mei-W68 ${ }^{1}$ (dmspo11-deficient) homozygotes (from $26 \%$ to $87 \%$ ). However, the expression of the plant SPO11 cDNAs by the same promoter never rescued it (21-24\%; Table 2), and thus the DSBs introduced by a plant SPO11 in Drosophila are somehow different from those introduced by the host SPO11, Mei-W68 (DmSPO11). As mentioned in the Introduction, the SPO11 protein requires various species-specific interacting proteins for its meiotic function. Thus, it was expected that the expressed plant SPO11s, without their specific interacting proteins in Drosophila, would be unable to complement the meiotic defects due to the mei-W68 (dmspo11) mutation (see Discussion).

In meiotic recombination-defective mutants, the nondisjunction of homologous chromosomes in meiosis will theoretically generate an equal number of nullo-X eggs and diplo-X eggs (Figure 3B). A nullo-X egg fertilized with a single $\mathrm{X}$ sperm produces a special progeny called an $\mathrm{X} 0$ son, and a diplo- $\mathrm{X}$ egg fertilized with a single $\mathrm{Y}$ sperm produces a progeny called an XXY daughter, when tested females are crossed with normal males with $\mathrm{XY}$ chromosomes. The cross of the mei-W68 ${ }^{1}$ homozygous females without the transgene to wild-type males produced progeny that included $11.4 \%$ X0 sons, reflecting the proportion of nullo-X eggs to total fertile eggs, and $8.3 \% \mathrm{XXY}$ daughters, reflecting the proportion of diplo-X eggs to total fertile eggs (Table 3 ). We interpreted this disparity in the proportion of nullo-X eggs to diplo-X eggs to be the outcome of $\mathrm{X}$ chromosome loss caused by spontaneously generated DSBs, occurring with inappropriate timing relative to the progression of meiosis. This interpretation is consistent with the 7.4\% background level of DSB signals in mei-W68 ${ }^{1}$ homozygotes with mus301 DSB-repair defects. It is further supported by the fact that the X-ray irradiation of cells at meiosis efficiently rescued the crossing-over defect and the homologous chromosome nondisjunction in meiW68 female flies, while the irradiation of the premeiotic and postmeiotic cells did neither [48].

The expression of AtSPO11-2 significantly increased $\mathrm{X}$-chromosome loss, as observed by an increase in nullo-X eggs without associated diplo-X eggs (Table 3 ). The expression of AtSPO11-1 increased it slightly (Table 3 ). These results support the conclusion obtained from the above cytological assays that the expression of either AtSPO11-1 or AtSPO11-2 actually induces DSBs in the $\mathrm{X}$-chromosomes of oocytes in the absence of functional DmSPO11 in Drosophila, but the induced DSBs are not repaired by the normal recombination required for chiasma formation. The DSB forming activity of AtSPO11-1 and AtSPO11-2, as shown by cytological and genetic means, was independent of other plant proteins, such as Arabidopsis PRD1, indicating that AtSPO11-1 and AtSPO11-2 express the DSB forming activity either by themselves or with the use of functional Drosophila analogues of other plant proteins.

\section{Identification of a novel SPO11-homologue}

Since the bioassay for DSB induction by an expressed SPO11 transgene successfully detected the DSB-forming activities of AtSPO11-1 and AtSPO11-2, we then tried to identify the SPO11s that function in DSB formation in the rice O. sativa. Candidates of rice SPO11-homologues were cloned from a cDNA library, constructed from RNA isolated from anthers containing meiotic pollens of japonica rice, Nipponbare (see Materials and Methods). OsSPO11A, OsSPO11B and OsSPO11C are almost the same as OsTOP6A, OsTOP6B and OsTOP6C, respectively, of indica rice [20]. The fourth rice SPO11-homologue, AK101363, was found by a homologous sequence search of the database of fulllength cDNA clones from japonica rice, KOME (Knowledge-based Oryza Molecular Biological Encyclopedia; http://cdna01.dna.affrc.go.jp/cDNA/), using the amino acid sequence of AtSPO11-1, and consists of 487 amino acid residues.

We constructed the full-length cDNAs of all rice SPO11 candidates from the cloned cDNA fragments (see Materials and Methods). Consistent with 


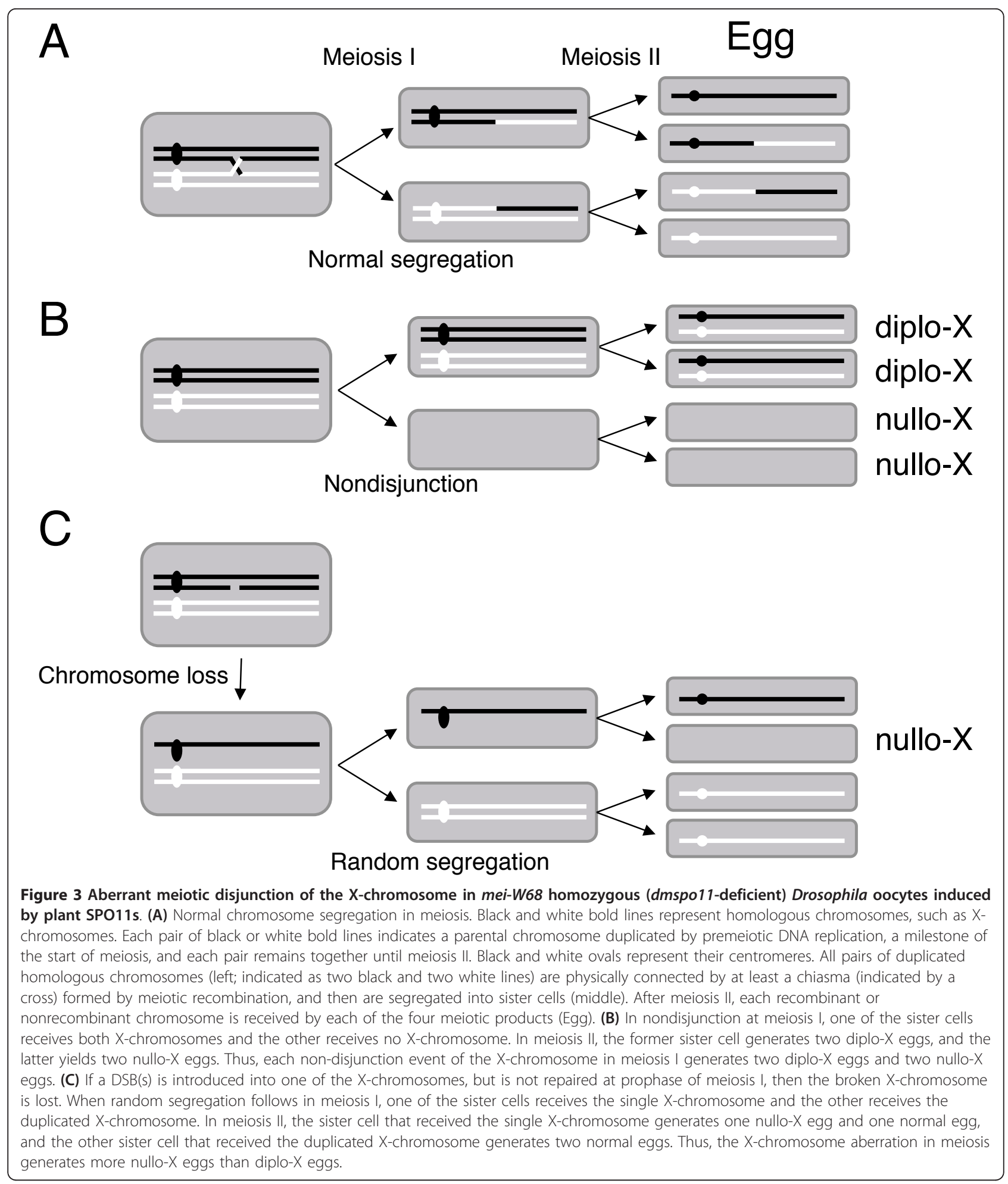

OsSPO11A, OsSPO11B and OsSPO11C, the amino acid sequence derived from the fourth SPO11-homologue contained all five conserved motifs of SPO11, as well as the conserved tyrosine (tyrosine 135 of S. cerevisiae) that is assumed to be essential for the DSB-forming activity in the first motif (Figure 4A) [5,49]. The DxD sequence in motif $\mathrm{V}$, which is proposed to coordinate a $\mathrm{Mg}^{2+}$ cation [49], is also conserved in the fourth SPO11-homologue. Thus, we tentatively named the fourth SPO11-homologue OsSPO11D (DDBJ accession 
Table 2 Effects of plant SPO11 expression on fertility in the Drosophila mei-W68 ${ }^{1}$ (dmspo11-deficient) homozygote

\begin{tabular}{|c|c|c|c|c|c|}
\hline $\begin{array}{l}\text { mei-W68 }{ }^{1} \text { hetero- } \\
\text { zygosity }\end{array}$ & Transgene & Mothers crossed & Total eggs laid & Pupae generated & $\%$ Fertility \\
\hline$+/ 1$ & none & 37 & 3768 & 3309 & 88 \\
\hline $1 / 1$ & none & 107 & 6109 & 1568 & 26 \\
\hline $1 / 1$ & $m e i-W 68^{+} \S$ & 11 & 1207 & 1047 & 87 \\
\hline $1 / 1$ & AtSPO11-1 $1^{\mathrm{a}}$ & 40 & 2880 & 691 & 24 \\
\hline $1 / 1$ & 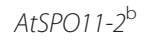 & 20 & 1536 & 359 & 23 \\
\hline $1 / 1$ & OsSPO11D & 60 & 3577 & 753 & 21 \\
\hline
\end{tabular}

Every female $(w / w)$ was crossed to a few males of Canton-S strain $\left(w^{+} / Y\right)$. The numbers of total eggs laid and the pupae subsequently generated were scored. Fertility was calculated by dividing the number of pupae (representing viable eggs) by the number of total eggs. Transgenes are indicated as Vector nameigene, insertion \#\}.

§: P\{hsp83-mei-W68 $8^{+}$cDNA, M53-3\}

a: P\{hsp83-AtSP011-1 cDNA, A5-1\}

b: P\{hsp83-AtSPO11-2 cDNA, 2M3-1\}

c: $P\{h s p 83-O s S P 011 D$ cDNA, F2-1\}

No. AB219540). While OsSPO11A, OsSPO11B and OsSPO11C are very likely to correspond to AtSPO11-1, AtSPO11-2 and AtSPO11-3, respectively, with similarities of 58\%, 63\% and 69\%, OsSPO11D has no Arabidopsis counterpart, since it shows low similarity to the Arabidopsis SPO11s (20 to 25\%), and a BLAST search revealed no similar gene among the genomes of other plants, including Sorghum [50] and Brachypodium [51], which are both phylogenetically much closer to rice than Arabidopsis. Thus, SPO11D is unique to rice.

Figure 4B shows the schematic genomic structures of the four rice SPO11-homologues, which were obtained from the rice genomic sequence in the TIGR rice genome database, using the full-length cDNA sequences as queries. The gene encoding the novel fourth SPO11- homologue, OsSPO11D, is 1,572 bp in length and consists of two exons and an intron. This gene structure with few introns is similar to that of OsSPO11C, but differs from those of OsSPO11A and OsSPO11B, which have more than ten exons and introns. We derived the amino acid sequences of the SPO11s of animals, plants, and fungi and the archaeal topoisomerase VI subunit A (TOP6A) from the NCBI web site (Additional File 1, Figure S1). Considering the property that the functionally less important molecules or parts of a molecule evolve faster than the more important ones [52], we focused on Motifs I to V of the SPO11-homologues for phylogenetic analyses by CLUSTALX, and the results are illustrated as a phylogenetic tree (Figure 4C). The previously reported SPO11-homologues of Arabidopsis

Table 3 Effects of plant SPO11 expression on X chromosome nondisjunction in the Drosophila mei-W68 ${ }^{1}$ homozygote

\begin{tabular}{|c|c|c|c|c|c|c|}
\hline $\begin{array}{l}\text { Transgene of mei-W68 } \\
\text { homozygote }\end{array}$ & $\begin{array}{l}\text { Mothers } \\
\text { crossed }\end{array}$ & $\begin{array}{l}\text { Total progeny } \\
\text { scored }\end{array}$ & $\begin{array}{l}\text { Nullo-X } \\
\text { eggs } \\
\text { (\%) }\end{array}$ & $\begin{array}{l}\text { Diplo-X } \\
\text { eggs } \\
\text { (\%) }\end{array}$ & $\begin{array}{l}\text { Nullo-X eggs without associated } \\
\text { diplo-X eggs } \\
\text { (\%) }\end{array}$ & $\begin{array}{l}P \text { value from } \\
\text { chi test }\end{array}$ \\
\hline \multicolumn{7}{|l|}{ Exp. 1} \\
\hline None & 99 & 1283 & $146(11.4)$ & $106(8.3)$ & $40(3.1)$ & - \\
\hline AtSPO11-1 $1^{\mathrm{a}}$ & 74 & 1204 & $138(11.5)$ & $95(7.9)$ & $43(3.6)$ & $5.3 \mathrm{E}-01$ \\
\hline AtSPO11-2 $2^{b}$ & 71 & 1187 & $178(15.0)$ & $88(7.4)$ & $90 * *(7.6)$ & $6.9 \mathrm{E}-07$ \\
\hline OsSPO11D & 97 & 1384 & $184(13.3)$ & $106(7.7)$ & $78 * *(5.6)$ & $1.6 \mathrm{E}-03$ \\
\hline \multicolumn{7}{|l|}{ Exp. 2} \\
\hline none & 55 & 1245 & $119(9.6)$ & $88(7.1)$ & $31(2.5)$ & - \\
\hline OsSPO11D & 57 & 1994 & $175(8.8)$ & $129(6.5)$ & $46(2.3)$ & 7.4E-01 \\
\hline$m e i-W 68^{+e}$ & 22 & 3434 & $3(0.09)$ & $3(0.09)$ & $0(0)$ & - \\
\hline
\end{tabular}

Every female $(w / w)$ was crossed to a few males of Canton-S strain $\left(w^{+} / Y\right)$. Nullo-X and diplo-X eggs were detected as $w^{+}$sons $(\mathrm{X} 0)$ and as $w$ daughters $(\mathrm{XXY})$, respectively. The number of nullo-X eggs without associated diplo-X eggs was calculated by subtracting the number of diplo- $X$ eggs from the number of nullo- $X$ eggs. The number of diplo-X eggs was used as the expected value for the number of nullo-X eggs with associated diplo-X eggs, which were generated by chromosome nondisjunction during the first meiotic division. The egg laying periods were 5 days and 10 days in Exp. 1 and Exp. 2 , respectively. Transgenes are indicated as Vector name\{gene, insertion \#\}.

a: $P\{h s p 83-A t S P 011-1$ cDNA, A5-1\}

b: P\{hsp83-AtSP011-2 cDNA, 2M3-1\}

c: $P\{h s p 83-O s S P 011 D$ cDNA, F2-1\}

$\mathrm{d}$ : P\{hsp83-OsSPO11D ${ }^{\text {Y213F }}$, F22-F2\}

e: $P\left\{\right.$ hsp83-mei-W68 ${ }^{+}$cDNA, M53-3\}

***: $P<1 \mathrm{E}-03$; **: $1 \mathrm{E}-03<P<1 \mathrm{E}-02$; $: 1 \mathrm{E}-02<P<5 \mathrm{E}-02$ 

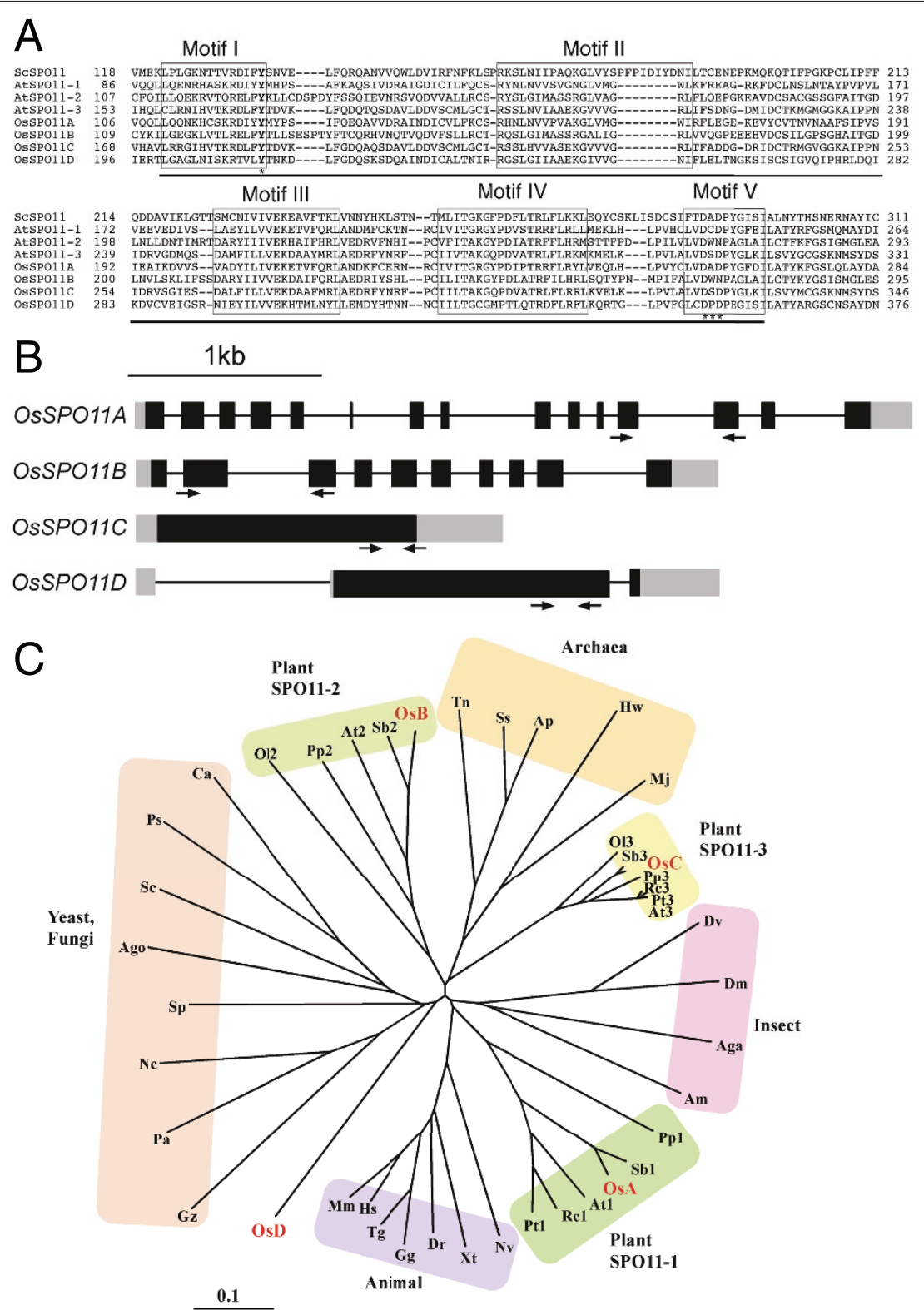

Figure 4 Comparative analysis of SPO11-homologues. (A) Multiple sequence alignment of motifs I to V of SPO11. The bold tyrosine (Y) residue with an asterisk in motif $\mathrm{I}$ is the putative active center for covalent bonding to the $5^{\prime}$ termini of cleaved DNA upon meiotic doublestranded cleavage. The triple asterisk in motif $\mathrm{V}$ indicates the conserved DXD sequence (see text). Underlined sequences were used for phylogenetic analyses. (B) Schematic diagrams of the genomic structures of OsSPO11 genes. Black boxes, coding regions; grey boxes, untranslated regions; solid lines, introns. Arrows indicate primers for real-time RT-PCR analyses. (C) Phylogenetic analyses of the amino acid sequences of motifs I-V. A neighbor-joining (NJ) tree was constructed using ClustalX ver. 2.0. Aga, Anopheles gambiae (EAA05541); Ago, Ashbya gossypii (AAS51945); Am, Apis mellifera (XP_001122679); Ap, Aeropyrum pernix (BAA79679); At1, Arabidopsis thaliana (CAB81544); At2, A. thaliana (CAB81545); At3, A. thaliana (ABI54341); Ca, Candida albicans (EAK95423); Dm, Drosophila melanogaster (AAC61735); Dr, Danio rerio (NP_991245); Dv, Drosophila virilis (EDW61133); Gg, Gallus gallus (XP_001232076); Gz, Gibberella zeae (XP_386125); Hs, Homo sapiens (AAD52562); Hw, Haloquadratum walsbyi (CAJ52765); Mj, Methanocaldococcus jannaschii (Q57815); Mm, Mus musculus (AAD52563); Nc, Neurospora crassa (CAB88597); Nv, Nematostella vectensis (EDO44118); Ol2, Ostreococcus lucimarinus (ABO99188); Ol3, O. lucimarinus (ABO95960); OsA, OsSPO11A; OsB, OsSPO11B; OsC, OsSPO11C; OsD, OsSPO11D; Pa, Podospora anserina (CAP73361); Pp1, Physcomitrella patens (EDQ80601); Pp2, P. patens (EDQ56207); Pp3, P. patens (EDQ71569); Ps, Pichia stipitis (XP_001384151); Pt1, Populus trichocarpa (EEE85181); Pt3, P. trichocarpa (EEE71999); Rc1, Ricinus communis (EEF39612); Rc3, R. communis (EEF37163); Sb1, Sorghum bicolor (EER93438); Sb2, S. bicolor (EES14573); Sb3, S. bicolor (EER95053); Sc, Saccharomyces cerevisiae (AAA65532); Sp, Schizosaccharomyces pombe (CAB11511); Ss, Sulfolobus shibatae (CAA71605); Tg, Taeniopygia guttata (XP_002195778); Tn, Thermoproteus neutrophilus (ACB40317); Xt, Xenopus tropicalis (AAH80352). The entire sequences of these SPO11 homologues are shown in Additional File 1 Figure S1. 
and rice were divided into three phylogenetic groups [53]. OsSPO11D is outside the three groups, and rather close to the fungal SPO11s (Figure 4C). This result suggests the unique origin of OsSPO11D, among the rice SPO11-homologues.

\section{OsSPO11D is expressed in anthers containing meiotic pollen mother cells}

We then examined the expression of the rice genes encoding SPO11-homologues at the mRNA level, in anthers containing different developmental stages of pollens, from meiotic to mature pollens, as well as in leaves and roots, by the use of quantitative real-time RT-PCR. In rice, the distance between the auricles of the last two leaves (DALL) correlates well with the developmental stage of pollens. The panicle in the $0 \mathrm{~cm}$ DALL (stage A0) contains the majority of pollen mother cells at meiosis [54]. The panicle in the $10 \mathrm{~cm}$ DALL (stage A10), which is almost two days before heading, contains the mature pollens. The flowers at the BB stage (before blooming) are one day before blooming. The panicles in the $3 \mathrm{~cm}$ DALL (stage A3) and $5 \mathrm{~cm}$ DALL (stage A5) contain immature to young mature pollens. We isolated the anthers from Nipponbare panicles at the different stages according to the DALL size, and analyzed the expression of the four genes. The results are shown in Figure 5.

The expression patterns of OsSPO11A and OsSPO11B were very similar to each other and rather different from those expected from their meiosis-specific functions; i.e., the OsSPO11A and OsSPO11B genes showed lower and constant levels (2-4 fold) of expression in the anthers containing pollens of all stages (A0 to A10), from meiosis to mature pollen, than OsSPO11C and OsSPO11D, although their expression levels were higher than those in the somatic cells, such as leaves and roots. The expression of OsSPO11A and OsSPO11B was induced to a 3-4 fold higher level (10-26 fold of the level in leaves) only at the $\mathrm{BB}$ stage, as compared to the other stages of pollen development.

The amounts of OSSPO11C mRNA during pollen development from meiosis to the mature pollen stage were within 2.5-fold of that in leaves, and tended to decrease through pollen development, but were generally (except in roots) higher than those of the other SPO11 genes, except for OsSPO11D. These OsSPO11C expression profiles suggest that it might be expressed in the somatic cells of leaves and anthers.

On the other hand, the expression pattern of the OsSPO11D mRNA resembled those of genes that are specifically expressed in meiosis; i.e., the amount of OsSPO11D mRNA increased to a much higher level ( $c a$. 20 -fold higher than the level in leaves) in the anthers containing meiotic pollens at the $0 \mathrm{~cm}$ DALL (stage
$\mathrm{A} 0$ ), and subsequently decreased. In the anthers containing meiotic pollens, the amount of OsSPO11D mRNA was the highest among the OsSPO11 genes; i.e., the amount was 4-6-fold higher than those of OsSPO11A and OSSPO11B (Figure 5). These results support the proposal that, unlike the other OsSPO11 homologues, the transcription of OSSPO11D is induced specifically and transiently in the meiotic stage of pollen development, suggesting that OsSPO11D plays an important role in rice meiosis.

\section{OsSPO11D has DSB-forming activity, as detected by the Drosophila bioassay}

To investigate whether OsSPO11D, OsSPO11A and OsSPO11B can induce DSBs, we measured the DSB forming activity by each of their transgenes, expressed under the control of the Drosophila hsp 83 promoter, in the mei-W68 (dmspo11) mus301 (DSB-repair defective) double mutant flies. The expression of OsSPO11D in the flies generated large numbers of oocyte nuclei with both kinds of DSB signals, the $\gamma-\mathrm{H} 2 \mathrm{Av}$ signals and the defective karyosome morphology (Figures $1 G$ and 2). In order to confirm that OsSPO11D directly introduced DSBs in Drosophila oocytes, we expressed a mutant transgene, OsSPO11D ${ }^{Y 213 F}$, with the phenylalanine-substitution of the 213th tyrosine in the putative catalytic center, under the control of the Drosophila hsp83 promoter. The OsSPO11D ${ }^{Y 213 F}$ transgenes were expressed at a similar level as the wild-type OSSPO11D transgene (Table 1), but never showed any additional DSB activity over the background level, in terms of DSB signals (Figure 2A) and karyosome morphology (Figure 2B). Thus, we conclude that OsSPO11D has intrinsic DSB-forming activity.

The expression of OsSPO11A in flies generated almost the same levels of DSB signals and defective karyosomes as AtSPO11-2 (Figures 1E, 1F and 2). On the other hand, the expression of OsSPO11B in flies did not generate any significant differences in the DSB signals and karyosome morphology over the negative control (without transgene), unlike the case of its Arabidopsis counterpart, AtSPO11-2 (Figure 2). In addition, the coexpression of OsSPO11A and OsSPO11B in flies showed no additive or synergistic effect on the generation of DSB-signals in fly oocytes (Figure 2). These results indicate that OsSPO11A and OsSPO11D have the ability to induce DSBs without the coexpression of other plant proteins in Drosophila oocytes.

To confirm the DSB activity of OsSPO11D in Drosophila, the nondisjunction test was performed. The expression of OsSPO11D in the mei-W6 $8^{1}$ homozygous (dmspo11-deficient) mus301-proficient flies increased the proportion of nullo-X eggs without associated diplo$\mathrm{X}$ eggs by $5.6 \%$ from the control level (3.1\%; Table 3; P 


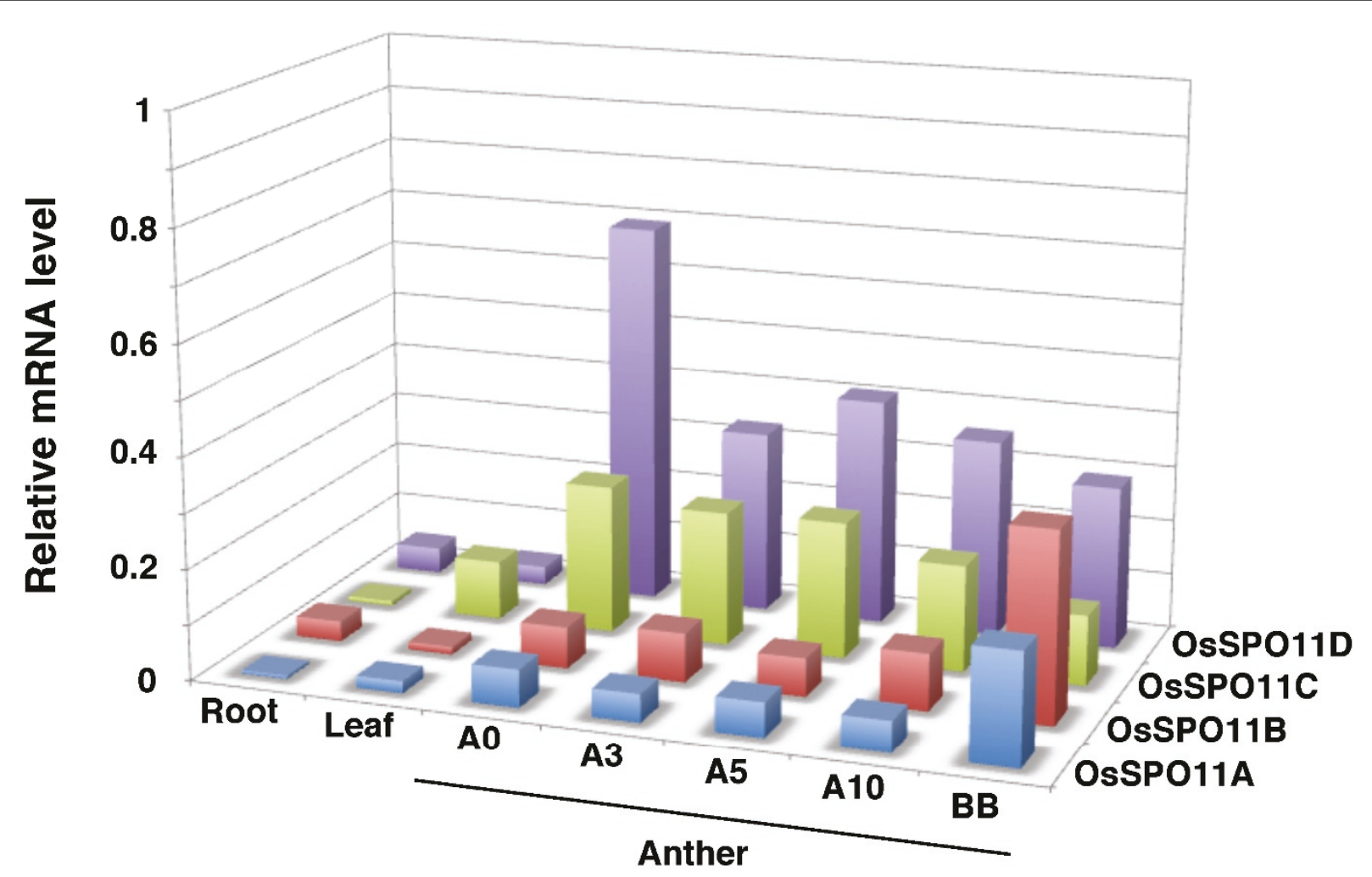

\begin{tabular}{|l|c|c|c|c|}
\hline & SPO11A & SPO11B & SPO11C & SPO11D \\
\hline Root $^{\star}$ & $0.005 \pm 0.001$ & $0.037 \pm 0.009$ & $0.008 \pm 0.004$ & $0.046 \pm 0.018$ \\
\hline Leaf & $0.021 \pm 0.002$ & $0.014 \pm 0.006$ & $0.106 \pm 0.017$ & $0.033 \pm 0.004$ \\
\hline A0 & $0.068 \pm 0.024$ & $0.075 \pm 0.027$ & $0.268 \pm 0.041$ & $0.695 \pm 0.263$ \\
\hline A3 & $0.051 \pm 0.037$ & $0.089 \pm 0.033$ & $0.242 \pm 0.122$ & $0.330 \pm 0.104$ \\
\hline A5 & $0.063 \pm 0.032$ & $0.069 \pm 0.025$ & $0.247 \pm 0.165$ & $0.410 \pm 0.192$ \\
\hline A10 & $0.057 \pm 0.019$ & $0.103 \pm 0.030$ & $0.192 \pm 0.177$ & $0.356 \pm 0.064$ \\
\hline BB & $0.203 \pm 0.052$ & $0.343 \pm 0.082$ & $0.126 \pm 0.021$ & $0.293 \pm 0.101$ \\
\hline
\end{tabular}

Figure 5 Quantitative real-time RT-PCR analyses of the amounts of rice SPO11 mRNAs in cells during pollen-development and in organs. The data shown in the table, representing the accuracy of the data in this figure, were plotted in a 3-dimensional manner for a comparison of their expression profiles. Root, seven day-old root after germination; $A 0$, the anthers in $0 \mathrm{~cm}$ DALL (stage A0); $A 3$, the anthers in 3 $\mathrm{cm}$ DALL (stage A3); $\mathrm{A} 5$, the anthers in $5 \mathrm{~cm}$ DALL (stage A5); A10, the anthers in $10 \mathrm{~cm}$ DALL (stage A10); BB, before blooming. All data obtained were normalized by the amount of actin gene (AK072796) mRNA, and are expressed as relative mRNA levels, which are the average values with SD obtained from three independent samples.

$<$ E-02 by chi test). The catalytic mutant transgene of OsSPO11D showed no increase in the proportion of nullo-X eggs without associated diplo-X eggs (Table 3 ). From these results, we conclude that OsSPO11D, like AtSPO11-1 and AtSPO11-2, has DSB forming activity.
Finally, to test whether the OsSPO11D-induced DSBs are repairable, we examined the DSB signals in meiW68-deficient, but mus301-proficient, flies with the OsSPO11D transgene under hsp83 promoter control $(P$ \{hsp83-OsSPO11D cDNA, F2-1\}). Among 116 oocyte 
nuclei (stages 2-8), only 2 nuclei showed $\gamma$-H2Av signals, and no defective karyosomes (stages 3-8) were observed (0/94), suggesting that the OsSPO11D-induced DSBs are joined by a DSB-repair mechanism that does not allow chiasma formation.

\section{Discussion}

Plants have multiple functional SPO11 candidates (see Introduction), and the SPO11 candidate(s) responsible for meiotic DSB formation has not been identified. To answer this question, we developed a Drosophila bioassay for the quantitative evaluation of the DSB activity of SPO11 candidates expressed from transgenes in fly oocytes. The interspecies bioassay that we developed in this study was effective to detect the DSB signals generated by the trans-expression of SPO11s and to identify the SPO11s with DSB-forming activities, among the candidates in plants. It was shown for the first time that both AtSPO11-1 and AtSPO11-2 exhibit DSB activity alone, in the absence of species-specific (i.e. Arabidopsis) interacting proteins for SPO11 functions. Arabidopsis has three genes encoding SPO11 homologues. Two of them (AtSPO11-1 and AtSPO11-2) are required for meiosis. Our results are surprising, since previous genetic studies showed that both AtSPO11-1 and AtSPO11-2 are required for meiosis, and interacting proteins, such as PRD1, are required for the SPO11 functions (see Introduction section). Note that our results do not mean that AtSPO11-1 and AtSPO11-2 work independently in Arabidopsis. It is likely that in Arabidopsis, SPO11-interacting proteins coordinate AtSPO11-1 and AtSPO11-2 to induce functional meiotic DSBs, leading to chiasma formation.

By using this method, we further investigated the three rice SPO11 homologues and a novel one, OsSPO11D. OsSPO11A and OsSPO11B are considered as the counterparts of Arabidopsis AtSPO11-1 and AtSPO11-2, respectively, from their amino acidsequence similarities. The bioassay revealed that OsSPO11A has DSB activity, as in the case of its Arabidopsis counterpart (Figure 2). However, we obtained another unexpected result from other tests: our assay did not detect any significant DSB-forming activity of OsSPO11B, unlike the case of its Arabidopsis counterpart, AtSPO11-2 (Figure 2). This was surprising, since OsSPO11B was expressed in the fly at a similar level as OsSPO11A, and at two- to five-fold higher levels than AtSPO11-1, AtSPO11-2 and OsSpo11D, which all showed DSB signals in the oocyte nuclei (Table 1 and Figure 2). This observation does not support the suggestion from the phylogenetic analyses (Figure 4) that OsSPO11B is an orthologue of AtSPO11-2. OsSPO11D displayed robust DSB activity (Figures 1G and 2).
All of the results obtained from the analysis of the $\gamma$ $\mathrm{H} 2 \mathrm{Av}$ signals were supported in parallel by the analysis of the frequencies of oocyte nuclei with defective karyosome morphology in the DSB-repair defective fly, which represent the ectopically expressed plant SPO11-induced DSBs in Drosophila oocyte nuclei (Figure 2).

In order to obtain additional evidence for the DSB activity of SPO11s, we performed genetic tests, using the dmspo11-deficient, but mus301-proficient (DSBrepair proficient), flies. We assumed that if the DSBs induced by a plant SPO11 are not repaired, then the DSBs caused the X chromosome loss in Drosophila oocytes, and if they are repaired by a normal recombination process leading to chiasma formation, then the DSBs restored the normal meiotic X chromosome disjunction. The expression of DmSPO11 from the transgene fully restored the meiotic deficiencies of the dmspo11 mutant flies (Table 2). The expression of the rice SPO11 transgenes, as well as the Arabidopsis SPO11s, in oocytes bearing the dmspo11 mutation caused an increase in nullo-X eggs, without associated diplo-X eggs (Table 3). The aberrant X-chromosome segregation, which was induced by the expression of the OsSPO11D, AtSPO11-1 and AtSPO11-2 transgenes in dmspo11 mutant flies, correlated well with the abilities of the expressed OsSPO11D, AtSPO11-1 and AtSPO112 proteins to induce DSBs, as shown by the $\gamma$-H2Av signals in the oocyte nuclei of the dmspo11 mus301 double mutant flies (Table 3 and Figure 2). This profile is explained by the $\mathrm{X}$ chromosome abnormalities caused by aberrant DSBs, as shown in Figure 3C. Therefore, we conclude that OsSPO11D, as well as AtSPO11-1 and AtSPO11-2, has DSB activity in oocytes.

Then, one may wonder why the DSBs induced by the plant SPO11s did not complement the meiotic defect of the dmspo11 mutant flies. The importance of the timing of DSB formation in meiotic disjunction was clearly shown by Bhagat et al. [48]: X-ray irradiation of prophase I oocytes of dmspo11-deficient mutant flies efficiently induced meiotic exchanges and suppressed meiotic nondisjunction of the mutant flies. However, pre-meiotic or post-meiotic irradiation did not induce meiotic exchange and caused more severe nondisjunction in the mutants. The S. cerevisiae Spo11 protein, expressed by the ubiquitous $A D H 1$ promoter in the host, exhibited DSB activity only in meiotic cells [55]. In addition, the DSB activity of Spo11 required meiosisspecific and meiosis-nonspecific interactors [56]. These findings indicated the presence of regulatory factors for Spo11 to exhibit the proper timing of the DSB activity. In this study, we found that DmSPO11, expressed by the ubiquitous hsp 83 promoter in the Drosophila dmspo11 mutant, allowed normal progression through meiosis. This suggested that, in Drosophila, a regulator 
conferring the stage specificity of DmSPO11-induced DSB formation prevents the DNA scission by the DmSPO11 protein in the premeiotic or postmeiotic stage, but in early pachynema, another regulatory factor induces the active form of the DmSPO11 complex, leading to DSB formation for crossing-over. It is likely that the Drosophila regulatory factors are unable to regulate the Arabidopsis SPO11s. Thus, the DSB induction by plant SPO11s, expressed by the ubiquitous promoter hsp 83 in the absence of plant SPO11-interacting protein factors, is unregulated and causes untimely DSBs in Drosophila oocytes. The DSBs induced at inappropriate times are repaired in the mus301-proficient fly as described, probably through inter-sister chromatidhomologous recombination or non-homologous endjoining, rather than inter-homologous chromosomerecombination, which is required for chiasma formation.

A recent paper reported that RNA interference for OsSPO11D (described as OsSPO11-2 in this paper) reduced pollen viability and seed setting rates in rice [57]. Our finding of the specific expression of OsSPO11D at the transcriptional level in the anthers containing meiotic pollens (Figure 5) further strengthens the specific role of OsSPO11D in meiotic recombination, as in the case of SPO11 in the yeast S. cerevisiae, which is expressed specifically at meiosis I under strict transcriptional regulation [58].

More studies on OsSPO11D are necessary to prove this hypothesis. The bioassay described in this study would also be helpful to identify species-specific SPO11interacting proteins that stimulate the SPO11 functions, the DSB forming activity specific for prophase I pachytene chromosomes, and the following interactions with repair enzymes. The biochemical characterization of purified SPO11s is necessary. We purified AtSPO11-1 [32], and other plant SPO11s (Y. S. unpublished observations) in soluble forms, but they lacked detectable endonuclease activity. A recent report claimed that OsSPO11D exhibited DSB forming activity by itself in vitro [57]. Further studies are needed to determine whether the purified SPO11s introduce the DSBs with either site- or sequence-specificity and are attached covalently at the termini of the cleavage sites, and which amino acid substitutions inactivate the SPO11s.

\section{Conclusions}

This study showed that, in the absence of other plant proteins, the Arabidopsis SPO11s required for meiosis, AtSPO11-1 and AtSPO11-2, and the rice AtSPO11-1 counterpart, OsSPO11A, exert the DSB activities by themselves or with the use of Drosophila functional analogues of other plant proteins. The Drosophila bioassay revealed a novel rice SPO11 homologue, OsSPO11D, which was suggested to be a functional SPO11 in rice meiosis, along with OsSPO11A.

\section{Methods}

Cloning of rice SPO11 CDNAs and plasmid constructions

Based on the DNA sequences of the Arabidopsis AtSPO11-1, AtSPO11-2 and AtSPO11-3 genes, four OsSPO11 gene candidates were identified from the rice cDNA database http://cdna01.dna.affrc.go.jp/cDNA/. The rice SPO11s corresponding to AtSPO11-1, AtSPO11-2 and AtSPO11-3 were named OsSPO11A, $O s S P O 11 B$ and OsSPO11C, respectively. The fourth OsSPO11 was designated as OsSPO11D. All OsSPO11s were amplified by RT-PCR, using RNA isolated from anthers containing meiotic pollens and the primers listed in Additional File 2, table S1. The OsSPO11C cDNA was cloned as a single DNA, and the other SPO11-homologue genes were amplified as two overlapping $5^{\prime}$ and $3^{\prime}$ fragments (Additional File 2, table $\mathrm{S} 1)$. The products were inserted into the pCR2.1 plasmid, using a TA Cloning ${ }^{\circledR}$ Kit (Invitrogen, Carlsbad, CA), and were sequenced. To obtain the whole genes, the EcoRI, AatII and ClaI restriction sites were used for OsSPO11A, $B$ and $D$, respectively, with the appropriate restriction sites on pCR2.1 (Additional File 2, table S1). To create the Drosophila transformants, we generated a P-vector bearing the Drosophila hsp 83 promoter, for the expression of the cloned gene in germ-line cells. The 0.9 -kb $h s p 83$ promoter genomic region was amplified by $\mathrm{PCR}$, using genomic DNA from the wild-type strain Canton-S and the following modified primers to create the XhoI (5') and HpaI (3') restriction sites: 5'-GGGCTCGAGG GGAACTTGAA GAAGTGCATA TTGGGG-3' and 5'-CCCGTTAACC AGACGCTGCT TGTTGTTACG ACGC-3', respectively [59]. The 0.9-kb XhoI/HpaI fragment was then purified. The P-vector pCasper-hs, which is generally used for heat-shock induced expression of transgenes, was digested with the XhoI and HpaI restriction enzymes to remove the $h s p 70$ promoter region, and the 8.4-kb XhoI/HpaI fragment was then ligated with the $0.9-\mathrm{kb} X h o \mathrm{I} / \mathrm{HpaI}$ fragment to form the new vector, pCasper-hsp83. The cDNAs encoding AtSPO11-1, AtSPO11-2, OsSPO11A, OsSPO11C, and OsSPO11D were re-amplified with the primers listed in Additional File 2, Table S1 and digested with BglII or BamHI and $K p n I$. These fragments were inserted into pCasperhsp83 at the BglII-KpnI sites. The mutant OsSPO11D cDNA, harboring the single amino acid substitution (Tyr 213 to Phe), was constructed by using a QuikChange Site-Directed Mutagenesis kit (Stratagene, La Jolla, CA, USA), and the primer pairs are listed in Additional File 2, table S1. 


\section{Genetic techniques}

Drosophila stock construction and maintenance were performed on standard cornmeal-molasses medium at room temperature $\left(22-25^{\circ} \mathrm{C}\right)$. All Drosophila crosses for tests were performed at $25^{\circ} \mathrm{C}$. Mutations and abbreviations are described in FlyBase [60]. The chromosomes used are as follows: the mutagen-sensitive mutant mus301 $1^{D 4}$, the deficiency uncovering the mus301 locus $D f(3 L) 66 C-G 28$, and the meiotic mutant mei-W68 ${ }^{1}$ (Bloomington Stock Center). The balancer chromosomes were $\mathrm{CyO}$ and TM3 (Kyoto Stock Center).

\section{Drosophila cytological techniques for ovary fixation and immunofluorescence}

Virgin females were aged for 4 to 5 days at $25^{\circ} \mathrm{C}$, and then their ovaries were dissected in $1 \times$ PBS. The ovaries were transferred to a microcentrifuge tube containing $4 \%$ paraformaldehyde in $1 \times \mathrm{PBS}$ and fixed for 30 min to $1 \mathrm{hr}$ at room temperature. The fixative was carefully removed, and the ovaries were washed in $0.5 \mathrm{ml}$ of CSC buffer (10 $\mathrm{mM}$ citric acid/sodium citrate buffer ( $\mathrm{pH}$ 6.0)) supplemented with $0.1 \%$ (v/v) Igepal CA-630 (Sigma no. 18896), which was used as a nonionic detergent in place of Triton $\mathrm{X}-100$. The ovaries were then denatured for $20 \mathrm{~min}$ at $85^{\circ} \mathrm{C}$, in $1.2 \mathrm{ml}$ of CSC buffer supplemented with $0.1 \%(\mathrm{v} / \mathrm{v})$ Igepal CA-630 [61]. The tissue was rinsed in $1 \mathrm{ml}$ of PBSI-BSA $(1 \times \mathrm{PBS}+0.1 \%$ Igepal CA-630 + 5\% BSA) and incubated at room temperature for $30 \mathrm{~min}$ with $10 \mu \mathrm{g} / \mu \mathrm{l}$ RNaseA in PBSI-BSA. The tissue was rinsed in PBSI-BSA, and then blocked for $1.5 \mathrm{hr}$ at room temperature in PBSI-BSA. Antibody staining was performed as described previously [62]. The first antibody, a mouse monoclonal anti-human phospho-H2AX (Ser139) antibody that cross-reacts with Drosophila $\gamma$-H2Av (1:100 dilution; Upstate Biotechnologies, Lake Placid, NY), was added in PBSI-BSA and incubated for $48 \mathrm{hrs}$ at $5^{\circ} \mathrm{C}$. The tissue was washed in PBSI $(1 \times$ PBS $+0.1 \%$ Igepal CA-630). The secondary antibody, donkey anti-mouse IgG conjugated with Alexa-555 (Molecular Probes, Eugene, OR), was diluted at 1:100 in PBSI-BSA and incubated for $16-24 \mathrm{hrs}$ at $5^{\circ} \mathrm{C}$. DNA staining was performed in the solution for the secondary antibody reaction, using OliGreen ${ }^{\mathrm{TM}}$ (Molecular Probes, Eugene, OR). Sample images were collected on a confocal microscope with a OLYMPUS FLUOVIEW FV1000, using the OLYMPUS FV10-ASW [Ver1.6] program, and adapted using the Photoshop 5.0 LE software (Adobe).

\section{Drosophila female fertility and X-chromosome nondisjunction assays}

For the determination of the female fertility and the $\mathrm{X}$ chromosome nondisjunction frequencies, each of the tested females carrying the white mutant allele on the
$\mathrm{X}$-chromosome was crossed to a few wild-type males with the white $^{+}$allele. After 2 days of egg laying, each cross was transferred to a new food vial every day. The number of eggs laid was scored, and subsequently, the number of pupae generated was scored. Fertility was determined by calculating (pupae)/(total eggs). X-chromosome nondisjunction produces nullo-X eggs and diplo-X eggs. Nullo-X eggs were detected as white ${ }^{+}$sons (X0 males) and scored. Diplo-X eggs were indicated by white mutant daughters (XXY females) and scored. The frequencies of nullo- $X$ eggs and diplo- $X$ eggs were calculated as the proportions of the numbers of $\mathrm{X} 0$ males and XXY females to total progeny, respectively.

\section{Quantitative real-time PCR of transgenes in Drosophila oocytes}

For the expression analysis of transgenes, ovary tissue was isolated from Drosophila. Total RNA was isolated by using Isogen (Nippon Gene, Tokyo, Japan). Quantitative real-time PCR was performed with CHROMO4 (Bio-Rad Laboratories, Hercules, CA, USA), using the PCR primers listed in Additional File 2, table S1.

\section{Phylogenetic tree}

Multiple alignments of the deduced amino acidsequences of SPO11-homologues were accomplished by using the ClustalX program [63]. The alignments were created with the program ClustalX 2.012, using the Gonnet series matrix (Gap Opening, 10; Gap Extension, 0.2; Delay Divergent Sequences, 30\%; Negative Matrix, off), and then were automatically converted to tree files with the Neighbor-Joining method. The results were visualized using the TREEVIEW software http://taxonomy.zoology.gla.ac.uk/rod/treeview.html.

\section{RNA extraction and quantitative real-time RT-PCR of SP011-homologues}

For the expression analysis of SPO11-homologues, panicles and leaves were harvested from rice plants (Oryza sativa L. var. Nipponbare) grown in pots under natural light conditions during the summer. Roots were harvested from Nipponbare seedlings grown on Murashige and Skoog's medium [64] for 7 days. The anthers were collected from each panicle, and the leaves and roots were frozen immediately in liquid nitrogen and then stored in a freezer $\left(-80^{\circ} \mathrm{C}\right)$ until use. Total RNA was isolated from anther, leaf and root tissues by using a TRIzol Plus RNA Purification kit (Invitrogen), according to the manufacturer's instructions. Reverse transcription was performed by using the Superscript ${ }^{\circledR}$ III first strand system (Invitrogen). For quantitative real-time RT-PCR experiments, PCR primers (19-24 bp, Additional File 2, table S1) were designed to amplify $70-118$ bp fragments with a melting point range from $75-85^{\circ} \mathrm{C}$, following 
Perkin-Elmer's recommendations for SYBR Green primers. Quantitative real-time RT-PCR was performed with an ABI Prism 7000 Sequence Detection System (Perkin-Elmer Applied Biosystems, Foster City, CA), using Power SYBR ${ }^{\circledR}$ Green PCR Master Mix (Applied Biosystems), in $25 \mu \mathrm{l}$ reaction mixtures containing the cDNA template and $50 \mathrm{nmol}$ primer. The standard twostep thermal cycling protocol was repeated 40 times, and then the melting points of the amplified products were determined with the prescribed dissociation protocol, to verify that the signal was derived from a single amplicon.

\section{Additional material}

Additional file 1: Supplementary Figure S1. Multiple alignments of conserved sequences of the SPO11 proteins for phylogenetic analyses. These alignments were constructed using the CLUSTALW program at DDBJ. Asterisks indicate invariant amino acids. For the species abbreviations, see the legend of Figure 4C.

Additional file 2: Supplementary Table S1: Primer sequences (5' to $\left.3^{\prime}\right)$

\section{List of abbreviations}

DSB: double-stranded break; RT-PCR: reverse transcriptase polymerase chain reaction; DALL: the distance between the auricles of the last two leaves; SD: standard deviation.

\section{Acknowledgements}

The authors thank Yasuko Kanno for technical assistance, Ayako Takahashi for constructing Drosophila transformants, and Tohru Miura, Hirokazu Nakatake and Michiyo Ando for assistance in this work. This research was supported by a grant from the Program for the Promotion of Basic Research Activities for Innovative Biosciences, Bio-oriented Technology Research Advancement Institution (BRAIN), and in part by a Grant-in-Aid from the Ministry of Education, Culture, Sports, Science and Technology of Japan to T. S. and K.K

\section{Author details}

'Cellular \& Molecular Biology Laboratory, RIKEN Advanced Science Institute, 2-1 Hirosawa, Wako, Saitama 351-0198, Japan. ²Department of Supramolecular Biology, Graduate School of Nanobioscience, Yokohama City University, Tsurumi-ku, Yokohama, Kanagawa 230-0045, Japan. ${ }^{3}$ Department of Agriculture, Tokyo University of Agriculture, Atsugi, Kanagawa 243-0034, Japan. ${ }^{4}$ Center for Genetic Resource Education \& Development, Kyoto Institute of Technology, Saga-Ippongi-cho, Ukyo-ku, Kyoto 616-8354, Japan. ${ }^{5}$ National Institute of Crop Science, 2-1-8 Kannondai, Tsukuba, Ibaraki 3058518, Japan.

\section{Authors' contributions}

YS performed the molecular genetic studies, including the construction of the full-length cDNAs and their expression vectors, assisted with the sequence alignment and helped to write the manuscript. TT performed the molecular genetic studies, especially the RT-PCR analysis of the rice SPO11 mRNAs, and assisted with the sequence alignment. YA performed cytological and genetic studies on Drosophila. KT and SA performed the molecular genetic studies on rice. MK and AK participated in the isolation of mRNA from rice meiotic tissues and their cDNA libraries. TM assisted with the design of the expression vectors of plant SPO11 CDNA and helped to write the manuscript. MY participated in the design of the Drosophila bioassay. KW assisted with the design of rice genetic experiments, performed their genetic analyses, and helped to write the manuscript, especially the part about the rice molecular genetic studies. TS participated in the design of the study and its coordination, and helped to write the manuscript. KK designed and performed the Drosophila bioassay, and participated in coordination and writing the manuscript. All authors read and approved of the final manuscript.

Received: 26 May 2011 Accepted: 16 January 2012

Published: 16 January 2012

\section{References}

1. Szostak JW, Orr-Weaver TL, Rothstein RJ, Stahl FW: The double-strandbreak repair model for recombination. Cell 1983, 33:25-35.

2. Nicolas A, Treco D, Schultes NP, Szostak JW: An initiation site for meiotic gene conversion in the yeast Saccharomyces cerevisiae. Nature 1989, 338(6210):35-39.

3. Sun H, Treco D, Schultes NP, Szostak JW: Double-strand breaks at an initiation site for meiotic gene conversion. Nature 1989, 338:87-90.

4. Keeney S, Giroux CN, Kleckner N: Meiosis-specific DNA double-strand breaks are catalyzed by Spo11, a member of a widely conserved protein family. Cell 1997, 88(3):375-384

5. Bergerat A, deMassy B, Gadelle D, Varoutas PC, Nicolas A, Forterre P: An atypical topoisomerase II from archaea with implications for meiotic recombination. Nature 1997, 386(6623):414-417

6. Nairz K, Klein F: mre11S-a yeast mutation that blocks double-strandbreak processing and permits nonhomologous synapsis in meiosis. Genes Dev 1997, 11(17):2272-2290.

7. Tsubouchi $\mathrm{H}$, Ogawa $\mathrm{H}$ : A novel mre11 mutation impairs processing of double-stranded breaks of DNA during both mitosis and meiosis. Mol Cell Biol 1998, 18:260-268.

8. Hartsuiker E, Mizuno K, Molnar M, Kohli J, Ohta K, Carr AM: Ctp1CtIP and Rad32Mre11 nuclease activity are required for Rec12Spo11 removal, but Rec12Spo11 removal is dispensable for other MRN-dependent meiotic functions. Mol Cell Biol 2009, 29(7):1671-1681.

9. Milman N, Higuchi E, Smith GR: Meiotic DNA double-strand break repair requires two nucleases, MRN and Ctp1, to produce a single size class of Rec12 (Spo11)-oligonucleotide complexes. Mol Cell Biol 2009, 29(22):5998-6005.

10. Keeney S, Kleckner N: Covalent protein-DNA complexes at the $5^{\prime}$ strand termini of meiosis-specific double-strand breaks in yeast. Proc Natl Acad Sci USA 1995, 92(24):11274-11278.

11. McKee $A H$, Kleckner $\mathrm{N}$ : A general method for identifying recessive diploid-specific mutations in Saccharomyces cerevisiae, its application to the isolation of mutants blocked at intermediate stages of meiotic prophase and characterization of a new gene SAE2. Genetics 1997, 146(3):797-816.

12. Lin $Y$, Smith GR: Transient, meiosis-induced expression of the rec6 and rec12 genes of Schizosaccharomyces pombe. Genetics 1994, 136(3):769-779.

13. McKim KS, Hayashi-Hagihara A: mei-W68 in Drosophila melanogaster encodes a Spo11 homolog: evidence that the mechanism for initiating meiotic recombination is conserved. Genes Dev 1998, 12(18):2932-2942.

14. Dernburg AF, McDonald K, Moulder G, Barstead R, Dresser M, Villeneuve AM: Meiotic recombination in $C$. elegans initiates by a conserved mechanism and is dispensable for homologous chromosome synapsis. Cell 1998, 94(3):387-398.

15. Cummings WJ, Celerin M, Crodian J, Brunick LK, Zolan ME: Insertional mutagenesis in Coprinus cinereus: use of a dominant selectable marker to generate tagged, sporulation-defective mutants. Curr Genet 1999, 36(6):371-382.

16. Metzler-Guillemain C, de Massy B: Identification and characterization of an SPO11 homolog in the mouse. Chromosoma 2000, 109(1-2):133-138.

17. Keeney S, Neale MJ: Initiation of meiotic recombination by formation of DNA double-strand breaks: mechanism and regulation. Biochem Soc Trans 2006, 34(Pt 4):523-525.

18. Hartung F, Puchta $\mathrm{H}$ : Molecular characterisation of two paralogous SPO11 homologues in Arabidopsis thaliana. Nucleic Acids Res 2000, 28(7):1548-1554

19. Hartung F, Puchta $\mathrm{H}$ : Molecular characterization of homologues of both subunits A (SPO11) and B of the archaebacterial topoisomerase 6 in plants. Gene 2001, 271(1):81-86 
20. Jain M, Tyagi AK, Khurana JP: Overexpression of putative topoisomerase 6 genes from rice confers stress tolerance in transgenic Arabidopsis plants. FEBS J 2006, 273(23):5245-5260.

21. Grelon M, Vezon D, Gendrot G, Pelletier G: AtSPO11-1 is necessary for efficient meiotic recombination in plants. EMBO J 2001, 20(3):589-600.

22. Stacey NJ, Kuromori T, Azumi Y, Roberts G, Breuer C, Wada T, Maxwell A, Roberts K, Sugimoto-Shirasu K: Arabidopsis SPO11-2 functions with SPO11-1 in meiotic recombination. Plant J 2006, 48(2):206-216

23. Hartung F, Angelis KJ, Meister A, Schubert I, Melzer M, Puchta H: An archaebacterial topoisomerase homolog not present in other eukaryotes is indispensable for cell proliferation of plants. Curr Biol 2002, 12(20):1787-1791.

24. Yin Y, Cheong H, Friedrichsen D, Zhao Y, Hu J, Mora-Garcia S, Chory J: A crucial role for the putative Arabidopsis topoisomerase VI in plant growth and development. Proc Natl Acad Sci USA 2002, 99(15):10191-10196.

25. Sugimoto-Shirasu K, Stacey NJ, Corsar J, Roberts K, McCann MC: DNA topoisomerase $\mathrm{VI}$ is essential for endoreduplication in Arabidopsis. Curr Biol 2002, 12(20):1782-1786

26. Yu H, Wang M, Tang D, Wang K, Chen F, Gong Z, Gu M, Cheng Z OsSPO11-1 is essential for both homologous chromosome pairing and crossover formation in rice. Chromosoma 2010, (Published online).

27. Keeney S: Mechanism and control of meiotic recombination initiation. Curr Top Dev Biol 2001, 52:1-53.

28. Bleuyard JY, Gallego ME, White Cl: Meiotic defects in the Arabidopsis rad50 mutant point to conservation of the MRX complex function in early stages of meiotic recombination. Chromosoma 2004, 113(4):197-203.

29. Puizina J, Siroky J, Mokros P, Schweizer D, Riha K: Mre11 deficiency in Arabidopsis is associated with chromosomal instability in somatic cells and Spo11-dependent genome fragmentation during meiosis. Plant Cell 2004, 16(8):1968-1978.

30. De Muyt A, Vezon D, Gendrot G, Gallois JL, Stevens R, Grelon M: AtPRD1 is required for meiotic double strand break formation in Arabidopsis thaliana. EMBO J 2007, 26(18):4126-4137.

31. Liu H, Jang JK, Kato N, MCKim KS: mei-P22 encodes a chromosomeassociated protein required for the initiation of meiotic recombination in Drosophila melanogaster. Genetics 2002, 162(1):245-258.

32. Shingu Y, Mikawa T, Onuma M, Hirayama T, Shibata T: A DNA-binding surface of SPO11-1, an Arabidopsis SPO11 orthologue required for normal meiosis. FEBS J 2010, 277(10):2360-2374.

33. Wu T-C, Lichten M: Meiosis-induced double-strand break sites determined by yeast chromatin structure. Science 1994 , 263(5146):515-518

34. Ohta K, Shibata T, Nicolas A: Changes in chromatin structure at recombination initiation sites during yeast meiosis. EMBO J 1994, 13(23):5754-5763.

35. Mizuno K-i, Emura Y, Baur M, Kohli J, Ohta K, Shibata T: The meiotic recombination hotspot created by the single base substitution, ade6M26, results in remodeling of chromatin structure in fission yeast. Genes Dev 1997, 11(7):876-886.

36. Madigan JP, Chotkowski HL, Glaser RL: DNA double-strand break-induced phosphorylation of Drosophila histone variant $\mathrm{H} 2 \mathrm{Av}$ helps prevent radiation-induced apoptosis. Nucleic Acids Res 2002, 30(17):3698-3705.

37. Staeva-Vieira E, Yoo S, Lehmann R: An essential role of DmRad51/SpnA in DNA repair and meiotic checkpoint control. EMBO J 2003, 22(21):5863-5874.

38. Jang JK, Sherizen DE, Bhagat R, Manheim EA, McKim KS: Relationship of DNA double-strand breaks to synapsis in Drosophila. J Cell Sci 2003, 116(Pt 15):3069-3077.

39. McCaffrey R, St Johnston D, Gonzalez-Reyes A: Drosophila mus301/spindle$C$ encodes a helicase with an essential role in double-strand DNA break repair and meiotic progression. Genetics 2006, 174(3):1273-1285.

40. Rogakou EP, Pilch DR, Orr AH, Ivanova VS, Bonner WM: DNA doublestranded breaks induce histone H2AX phosphorylation on serine 139. J Biol Chem 1998, 273(10):5858-5868.

41. Rogakou EP, Boon C, Redon C, Bonner WM: Megabase chromatin domains involved in DNA double-strand breaks in vivo. J Cell Biol 1999, 146(5):905-916.

42. Redon C, Pilch D, Rogakou E, Sedelnikova O, Newrock K, Bonner W: Histone H2A variants H2AX and H2AZ. Curr Opin Genet Dev 2002, 12(2):162-169.
43. Roig I, Liebe B, Egozcue J, Cabero L, Garcia M, Scherthan H: Female-specific features of recombinational double-stranded DNA repair in relation to synapsis and telomere dynamics in human oocytes. Chromosoma 2004, 113(1):22-33.

44. Cao $L$, Alani $E$, Kleckner $\mathrm{N}$ : A pathway for generation and processing of double-strand breaks during meiotic recombination in S. cerevisiae. Cell 1990, 61:1089-1101.

45. Baudat F, Nicolas A: Clustering of meiotic double-strand breaks on yeast chromosome III. Proc Natl Acad Sci USA 1997, 94(10):5213-5218.

46. Ghabrial A, Ray RP, Schupbach T: okra and spindle-B encode components of the RAD52 DNA repair pathway and affect meiosis and patterning in Drosophila oogenesis. Genes Dev 1998, 12(17):2711-2723.

47. Baker BS, Carpenter AT: Genetic analysis of sex chromosomal meiotic mutants in Drosophila melanogaster. Genetics 1972, 71(2):255-286.

48. Bhagat R, Manheim EA, Sherizen DE, McKim KS: Studies on crossoverspecific mutants and the distribution of crossing over in Drosophila females. Cytogenet Genome Res 2004, 107(3-4):160-171.

49. Nichols MD, DeAngelis K, Keck JL, Berger JM: Structure and function of an archaeal topoisomerase VI subunit with homology to the meiotic recombination factor Spo11. EMBO J 1999, 18(21):6177-6188.

50. Paterson AH, Bowers JE, Bruggmann R, Dubchak I, Grimwood J, Gundlach H, Haberer G, Hellsten U, Mitros T, Poliakov A, et al: The Sorghum bicolor genome and the diversification of grasses. Nature 2009, 457(7229):551-556

51. Vogel JP, Garvin DF, Mockler TC, Schmutz J, Rokhsar D, Bevan MW, Barry K, Lucas S, Harmon-Smith M, Lail K, et al: Genome sequencing and analysis of the model grass Brachypodium distachyon. Nature 2010, 463(7282):763-768.

52. Kimura M: The neutral theory of molecular evolution: a review of recent evidence. Japanese Journal of Genetics 1991, 66(4):367-386.

53. Malik SB, Ramesh MA, Hulstrand AM, Logsdon JM Jr: Protist homologs of the meiotic Spo11 gene and topoisomerase VI reveal an evolutionary history of gene duplication and lineage-specific loss. Mol Biol Evol 2007, 24(12):2827-2841.

54. Chen C-B, Xu Y-Y, Ma H, Chong K: Cell biological characterization of male meiosis and pollen development in rice. J Integr Plant Biol 2005, 47(6):734-744

55. Pecina A, Smith KN, Mezard C, Murakami H, Ohta K, Nicolas A: Targeted stimulation of meiotic recombination. Cell 2002, 111(2):173-184.

56. Arora C, Kee K, Maleki S, Keeney S: Antiviral protein Ski8 is a direct partner of Spo11 in meiotic DNA break formation, independent of its cytoplasmic role in RNA metabolism. Mol Cell 2004, 13(4):549-559.

57. An XJ, Deng ZY, Wang T: OsSpo11-4, a rice homologue of the archaeal TopVIA protein, mediates Double-Strand DNA cleavage and interacts with OsTopVIB. PLoS One 2011, 6(5):e20327.

58. Atcheson CL, DiDomenico B, Frackman S, Esposito RE, Elder RT: Isolation, DNA sequence, and regulation of a meiosis-specific eukaryotic recombination gene. Proc Natl Acad Sci USA 1987, 84(22):8035-8039.

59. Matsubayashi H, Yamamoto MT: REC, a new member of the MCM-related protein family, is required for meiotic recombination in Drosophila. Genes Genet Syst 2003, 78(5):363-371.

60. Grumbling G, Strelets V: FlyBase: anatomical data, images and queries. Nucleic Acids Res 2006, , 34Database: D484-488.

61. Shi SR, Imam SA, Young L, Cote RJ, Taylor CR: Antigen retrieval immunohistochemistry under the influence of $\mathrm{pH}$ using monoclonal antibodies. J Histochem Cytochem 1995, 43(2):193-201.

62. de Cuevas M, Lee JK, Spradling AC: alpha-spectrin is required for germline cell division and differentiation in the Drosophila ovary. Development 1996, 122(12):3959-3968.

63. Larkin MA, Blackshields G, Brown NP, Chenna R, McGettigan PA, McWilliam H, Valentin F, Wallace IM, Wilm A, Lopez R, et al: Clustal W and Clustal X version 2.0. Bioinformatics (Oxford, England) 2007, 23(21):2947-2948.

64. Murashige T, Skoog E: A revised medium for rapid growth and bioassay with tobacco tissue culture. Physiol Plant 1962, 15(3):473-497.

doi:10.1186/1471-2199-13-1

Cite this article as: Shingu et al:: The double-stranded break-forming activity of plant SPO11s and a novel rice SPO11 revealed by a Drosophila bioassay. BMC Molecular Biology 2012 13:1 